\title{
Region Based Level Set Segmentation of the Outer Wall of the Carotid Bifurcation in CTA
}

\author{
D. Vukadinovic*a, T. van Walsum ${ }^{\mathrm{a}}$, R. Manniesing ${ }^{\mathrm{a}}$, S.Rozie ${ }^{\mathrm{b}}$, A. van der Lugt ${ }^{\mathrm{b}}$, W.J. Niessen ${ }^{\mathrm{a}, \mathrm{c}}$ \\ ${ }^{a}$ Biomedical Imaging Group Rotterdam, Erasmus MC - University Medical Center Rotterdam \\ Departments of ${ }^{\mathrm{b}}$ Radiology and Medical Informatics, \\ ${ }^{c}$ Faculty of Applied Sciences, Delft University of Technology \\ Dr. Molewaterplein 50, 3015 GE Rotterdam, The Netherlands
}

\begin{abstract}
This paper presents a level set based method for segmenting the outer vessel wall and plaque components of the carotid artery in CTA. The method employs a GentleBoost classification framework that classifies pixels as calcified region or not, and inside or outside the vessel wall. The combined result of both classifications is used to construct a speed function for level set based segmentation of the outer vessel wall; the segmented lumen is used to initialize the level set. The method has been optimized on 20 datasets and evaluated on 80 datasets for which manually annotated data was available as reference. The average Dice similarity of the outer vessel wall segmentation was $92 \%$, which compares favorably to previous methods.
\end{abstract}

Keywords: Segmentation, Classification, Level set, Carotid, Outer vessel wall, Plaque, CTA

\section{INTRODUCTION}

Cardiovascular diseases are a main cause for morbidity and mortality worldwide [1]. One of the main causes of cardiovascular diseases is atherosclerosis, which can be effectively imaged with CT Angiography (CTA) [2]. The severity of stenosis caused by atherosclerosis in the carotid bifurcation is an important risk factor for stroke [3]; however, luminal stenosis as a parameter of carotid artery disease does not necessarily reflect the amount of atherosclerosis [4]. Quantification of plaque features may therefore provide a better diagnosis and prediction of cardiovascular events. This quantification requires segmentation of the lumen and outer vessel wall.

Many authors addressed the problem of outer wall segmentation and plaque quantification on both MRI and CT data. Olabarriaga et al. [5] proposed a deformable model based segmentation of the lumen and thrombus in abdominal aortic aneurysms in CTA data. For the wall segmentation a gray level modeling approach with a KNN classifier using intensity profiles sampled along the surface normal was used. The active shape model (ASM) based method by De Bruijne et al. [6], [7] also addressed aortic aneurysm segmentation. This method requires a manual delineation of the aneurysm in

the first slice after which the contour propagates to the adjacent slices based on grey value similarity. A slice-by-slice

control by the user is required. De Bruijne et al. [8] also proposed a 3D ASM which included a grey level appearance model which was based on non-parametric pattern classification. The user has to draw the top and bottom contours, and indicate the approximate aneurysm centre. Reported results are accurate, but the amount of interaction for initialization is still significant.

A snake based method for defining the vessel lumen and wall boundaries in MR images of the carotid artery was proposed by Yuan et al. [9]. Adams et al. [10] proposed a similar method, deforming two initial contours in MR carotid

artery images. Both methods require a high level of manual interaction. Adame et al. [11] proposed a gradient based ellipse fitting method combined with fuzzy clustering to outline the carotid artery outer vessel wall on MR images. The method requires manual interaction, namely a center point in the lumen, a seed point inside the lipid core and a circle that surrounds the vessel.

Liu et al. [12] proposed a method for carotid plaque segmentation in MRI using probability maps utilizing morphology information.

Medical Imaging 2011: Image Processing, edited by Benoit M. Dawant, David R. Haynor,

Proc. of SPIE Vol. 7962, 79623P - (C) 2011 SPIE - CCC code: 1605-7422/11/\$18 · doi: 10.1117/12.878114

Proc. of SPIE Vol. 7962 79623P-1 
In this paper a novel method for carotid artery outer vessel wall segmentation in CTA is presented, which is an extension of work we previously presented [13][14]. The main contribution of our work is that we combine voxel classification with a 3D level set segmentation for the carotid artery outer vessel wall rather than $2 \mathrm{D}$ ellipsoid fitting, which allows application of the method for segmenting the whole carotid bifurcation. Additionally, we evaluate the method proposed on a large number of CTA datasets.

A similar method has been reported for segmenting abdominal aortic aneurysms, where a support vector machine classifier is used to control a level set based segmentation. [15]

The remainder of this paper is organized as follows: In section 2 the methodology is presented. In section 3 the experimental set up is described followed by a discussion and conclusion in section 4 .

\section{METHODOLOGY}

\subsection{Overview}

The method consists of four steps. First, the vessel lumen is segmented using a level set approach initialized with three seed points indicated by the user in the CCA, ICA and external carotid artery (ECA); for detailed information on the lumen segmentation we refer to the work by Manniesing et al. [16]. Subsequently, using a set of image features, calcium objects which are part of the vessel wall, are detected using a GentleBoost classifier [13]. In the third step voxels are classified as within or outside the vessel, using the same classification method [13]. Then segmentation is performed by fitting a model to the classification image. In this work, a 3D region based level set method is applied which segments the outer boundary using the calcium and inner-outer vessel region classifications results to create a speed function. The classification process and the level set method are described in more detail below.

\subsection{Calcium and Vessel classification}

Calcified regions are important markers for determining the outer vessel wall location. As described in [13], the region of interest around lumen is thresholded with high threshold, $\mathrm{Th}=320 \mathrm{HU}$. In this way candidate objects are extracted which are further classified as true or false calcified regions. Classification is done based on a set of features, i.e. spatial, size, intensity and shape features [13].

For vessel classification, each pixel in the region of interest around lumen is described with a set of contextual image features extracted from radial profiles positioned radially from the lumen center. These features are used to classify each pixel as being inside or outside vessel wall [13].

As described in [13], calcium and inner-outer vessel region classification are combined by connecting lumen and calcified region since we are certain that this region is inside the vessel. In this way confidence image is created with negative values inside and positive values outside ranging from -1 to 1 (fig 1 ).

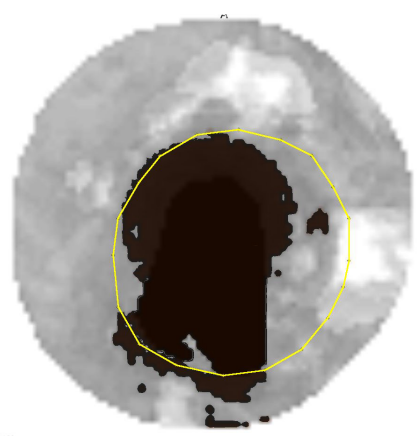

Fig. 1 An example of a classification result, showing the confidence image with darker regions more likely belonging to the inside of the outer vessel wall. The black mask highlights negative values in the image. The light, yellow contour represents the ground truth for the outer vessel wall as drawn by an experienced clinician. 


\subsection{Region based level set outer vessel wall segmentation}

Atherosclerotic plaque is usually present around the bifurcation. That is why it is essential that plaque is segmented in the region including bifurcation. Level sets are suitable for representing complex geometries. We therefore apply a region based level set [17] to segment the carotid artery outer vessel wall, using the the classification results.

The resulting image of the combined calcium and inner-outer vessel region classification represents the confidence that a pixel belongs to the vessel region (Fig 1): the confidence image values are negative inside and positive outside with values ranging between -1 and 1 . In case of a perfect classification, the vessel wall should pass through the zero values of the confidence image. However, simply thresholding the classification image will result in disconnected regions, and does not account for potential classification errors. Therefore, we use a region based level set segmentation to obtain one connected region with smooth boundaries. In the original paper of Chan and Vese on region based level set segmentations [17], the energy function that is minimized is defined as follows:

$$
F\left(c_{1}, c_{2}, C\right)=\mu \cdot \operatorname{Length}(C)+\lambda_{1} \int_{\text {inside }(C)}\left|i(x, y)-c_{1}\right|^{2} d x d y+\lambda_{2} \int_{\text {outside }(C)}\left|i(x, y)-c_{2}\right|^{2} d x d y,
$$

where

$$
\begin{aligned}
& C=\{(x, y) \in \Omega: \phi(x, y)=0\} \\
& \text { inside }(C)=\{(x, y) \in \Omega: \phi(x, y)>0\} \\
& \text { outside }(C)=\{(x, y) \in \Omega: \phi(x, y)<0\} \\
& \Omega \subset \mathbb{R}^{\mathrm{d}} \text { is the image domain and } i: \Omega->\mathbb{R}
\end{aligned}
$$

and $\mu \geq 0, \lambda_{1}, \lambda_{2} \geq 0$ are parameters, $i$ is a given image, $\mathrm{c}_{1}$ is the average intensity value of $i$ inside $\mathrm{C}$ and $\mathrm{c}_{2}$ the average value of $i$ outside $\mathrm{C}$.

In our case, we want to penalize positive values inside and negative values outside the zero level set. In order to achieve this, we choose constants $c_{1}$ and $c_{2}$ to be equally distant from zero with $c_{1}$ negative and $c_{2}$ positive. We choose $c_{1}=-0.5$ and $\mathrm{c}_{2}=0.5$ considering the values of $i$ image.

The speed function for the level set evolution is calculated by minimizing (1) with the Euler-Lagrange equation while taking into account the sign of image $i$ values inside and outside zero level set. The speed function for the confidence image then becomes:

$$
\frac{\partial \phi}{\partial t}=\delta(\phi)\left[\mu \operatorname{div}\left(\frac{\nabla \phi}{|\nabla \phi|}\right)+\lambda_{1}(i+0.5)^{2}-\lambda_{2}(i-0.5)^{2}\right]=0 \text { in }(0, \infty) \times \Omega,
$$

where $\phi$ is a level set function.

The above equation (2) is the implicit active contour model implemented in this paper. The lumen segmentation dilated with a "shape" structuring element of "size" pixels is used to initialize the level set.

\subsection{Plaque segmentation}

Using the carotid artery vessel wall and lumen segmented, different HU ranges selected to define different plaque components. The cut-off point for the distinction between calcifications and fibrous tissue was set at $130 \mathrm{HU}$, the value currently used for calcium scoring. The cut-off point for the distinction between fibrous tissue and lipid was set at $60 \mathrm{HU}$ as determined in previous studies [18]. We adjusted the cut-off point for the distinction between atherosclerotic plaque and vessel lumen for each patient on the basis of the full-width-half-maximum principle (mean lumen attenuation plus mean fibrous tissue attenuation (in our case the average was $88 \mathrm{HU}$ ) divided by two). The pixels surrounding the vessel lumen, with a density between $130 \mathrm{HU}$ and the adjusted cut-off value, were considered to be fibrous tissue. 
We implemented the carotid artery outer vessel wall segmentation using the ITK (Insight Tool Kit) implementation [19].

\section{EXPERIMENTS AND RESULTS}

\subsection{Data selection}

From a database containing CTA datasets with manually segmented carotid arteries, we randomly selected 20 datasets that had the common, internal and external carotid artery annotated. The CTA data were acquired on a MDCT scanner (Siemens, Sensation 16, Erlangen, Germany), with a slice thickness of $1.0 \mathrm{~mm}$ and a pixel size of $0.23 \mathrm{~mm}$.

\subsection{Parameter selection and evaluation}

The levelset integration time step was set to 0.01 . The remaining parameters of the method were curvature weight $\mu$ and the weights for inside and outside zero level set terms, $\lambda_{1}$, and $\lambda_{2}$. Pilot experiments showed that $\lambda_{2}$ should be equal or larger than $\lambda_{1}$, i.e. the negative values outside zero level set should be penalized more than positive values inside the zero level set. This was caused by the frequent occurrence of misclassified pixels inside the contour in the neighborhood of calcified regions (Fig 1). Note that this problem can not be addressed by increasing the curvature weight, as that would also result in misclassifying calcified regions. Additionally, from the pilot experiments it was concluded that the curvature term, $\mu$, should be smaller than $\lambda_{1}$ and $\lambda_{2}$.

The final levelset result is determined by the ratios between the three parameters, the magnitude of the parameters (while maintaining the ratios) only changes the convergence speed. We optimized level set parameters on 20 CTAs of carotid arteries by fixing $\mu$ to 1.0 and varied $\lambda_{1}$ and $\lambda_{2}$ from 1.0 to 6.2 in steps of 0.4 . Similarity indices are shown in figure 2 .

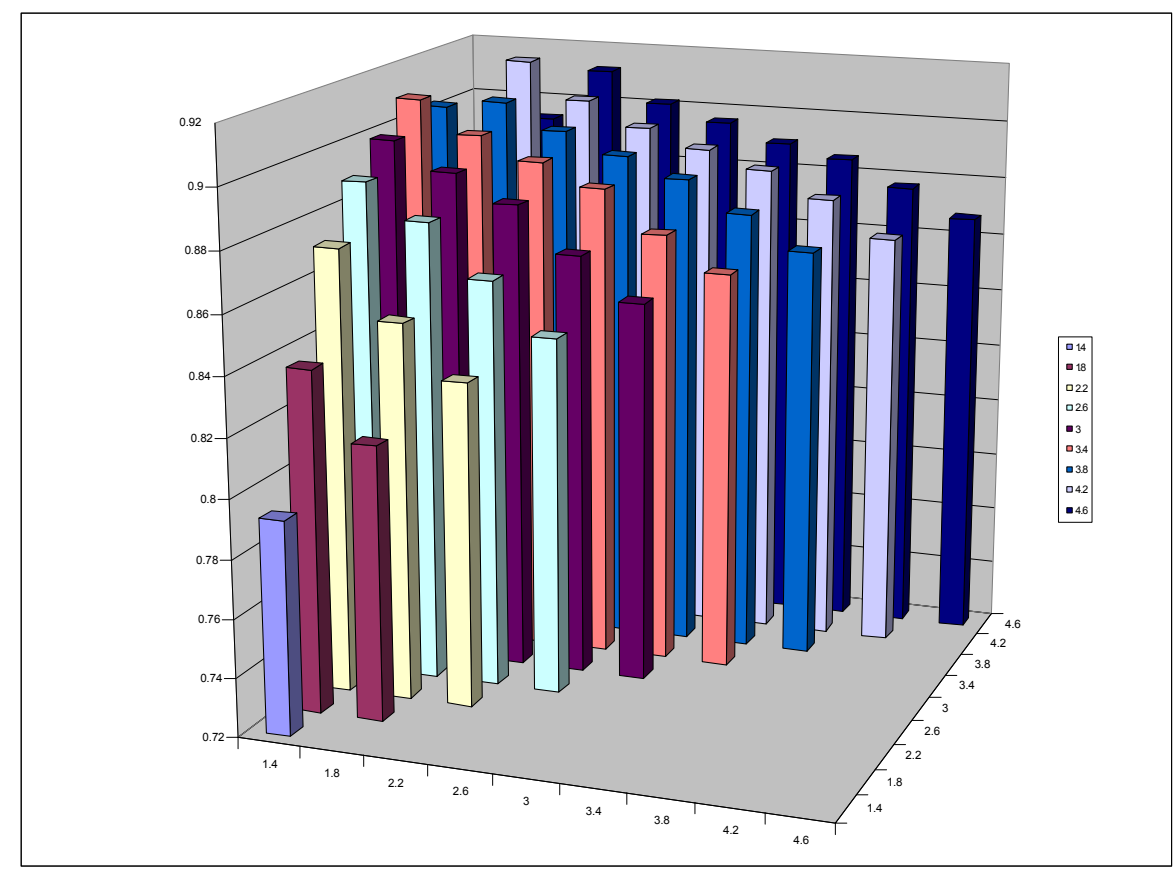

Fig. 2 Similarity indices between manual and automated segmentations of 20 CTAs of carotid arteries with $\mu=1$ and $\lambda_{1}$ and $\lambda_{2}$ ranging from 1 to 6.2 with 0.4 steps keeping $\lambda_{1}>\lambda_{2}$ 
Maximum Dice similarity index of $91.7 \%$ was reached for $\lambda_{1}=1.8$ and $\lambda_{2}=4.2$ and standard deviation was $2 \%$.

The average Dice similarity index over additional 80 datasets using optimized set of parameters was $91.61 \%$, and a standard deviation was 3\%. Example segmentations are shown in Fig. 3.
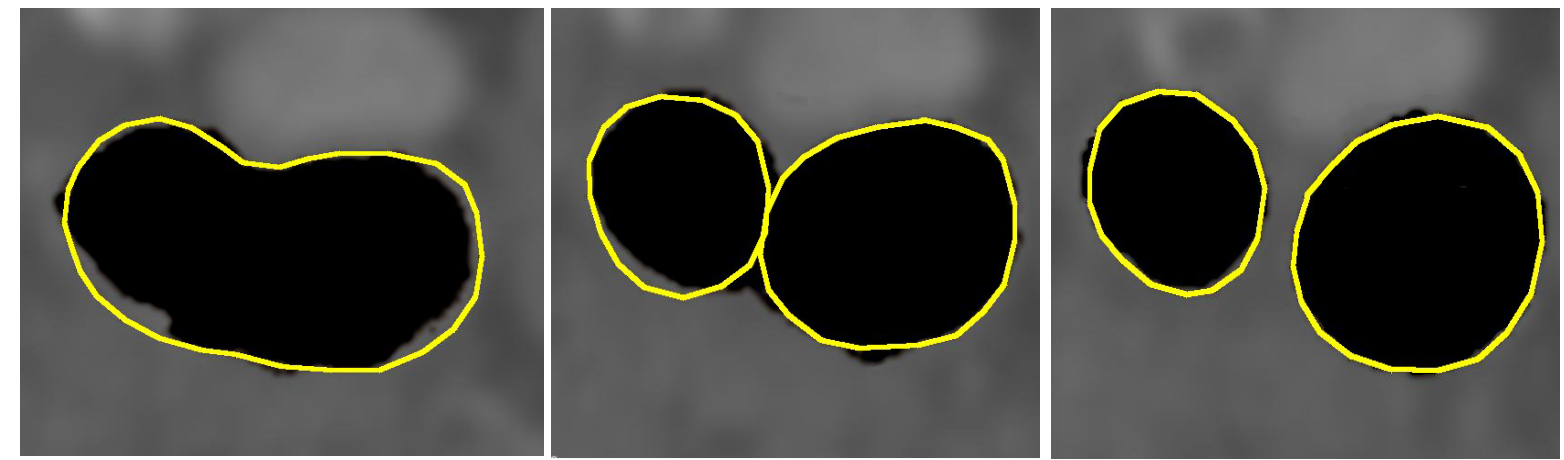

Fig 3. Three crossectional slices around carotid artery bifurcation. Black mask is segmentation by our method, yellow contour is the ground truth drawn by a clinician.

Plaque segmentation on 80 datasets reached an average similarity index of $80.43 \%$ with standard deviation of $6 \%$. An example of plaque components segmented on one cross-sectional slice with the automated method and manually is shown in fig. 4.
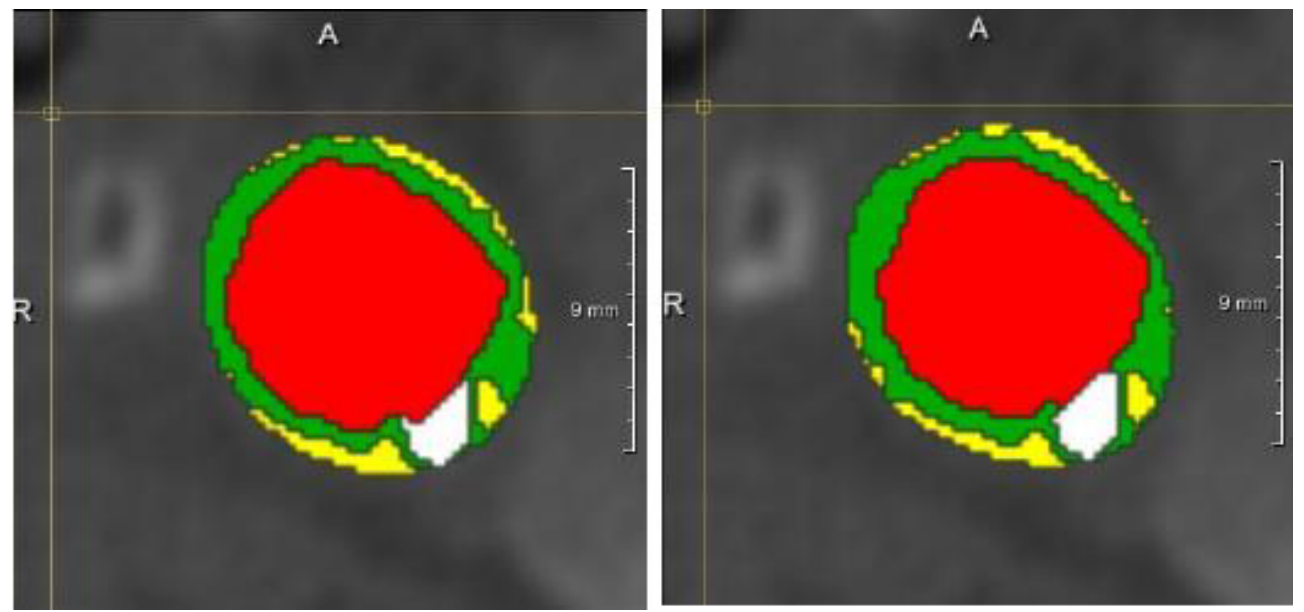

Fig. 4. Different plaque components on one crossectional slice segmented by the automated method (left) and manually (right). - lumen, $\square$ - fibrous, $\square$ - lipids, $\square$ - calcium.

Figure 5 shows areas of total plaque and different plaque components according to the manual observer and those derived from our automated method. The correlation coefficient between method and manual segmentation is low $\left(\mathrm{R}^{2}=\right.$ 0.46 ) in the case of lipid volume. This is probably die to the fact that lipid tissue is usually located at the boundary of the vessel wall and has small size. Therefore differences in outer vessel wall segmentation primarily lead to differences in the fibrous tissue and lipid class. Indeed, inter observer error is also especially high for the lipid area [14].

Correlation coefficients between manual and automated plaque and plaque components volumes are similar for the proposed method to the ones we previously achieved with 2D ellipse fitting on 5 datasets [14]. However, similarity 
between automated and manual measurement of total plaque area is much higher for the new method ( $73 \%$ compared to $81 \%)$.
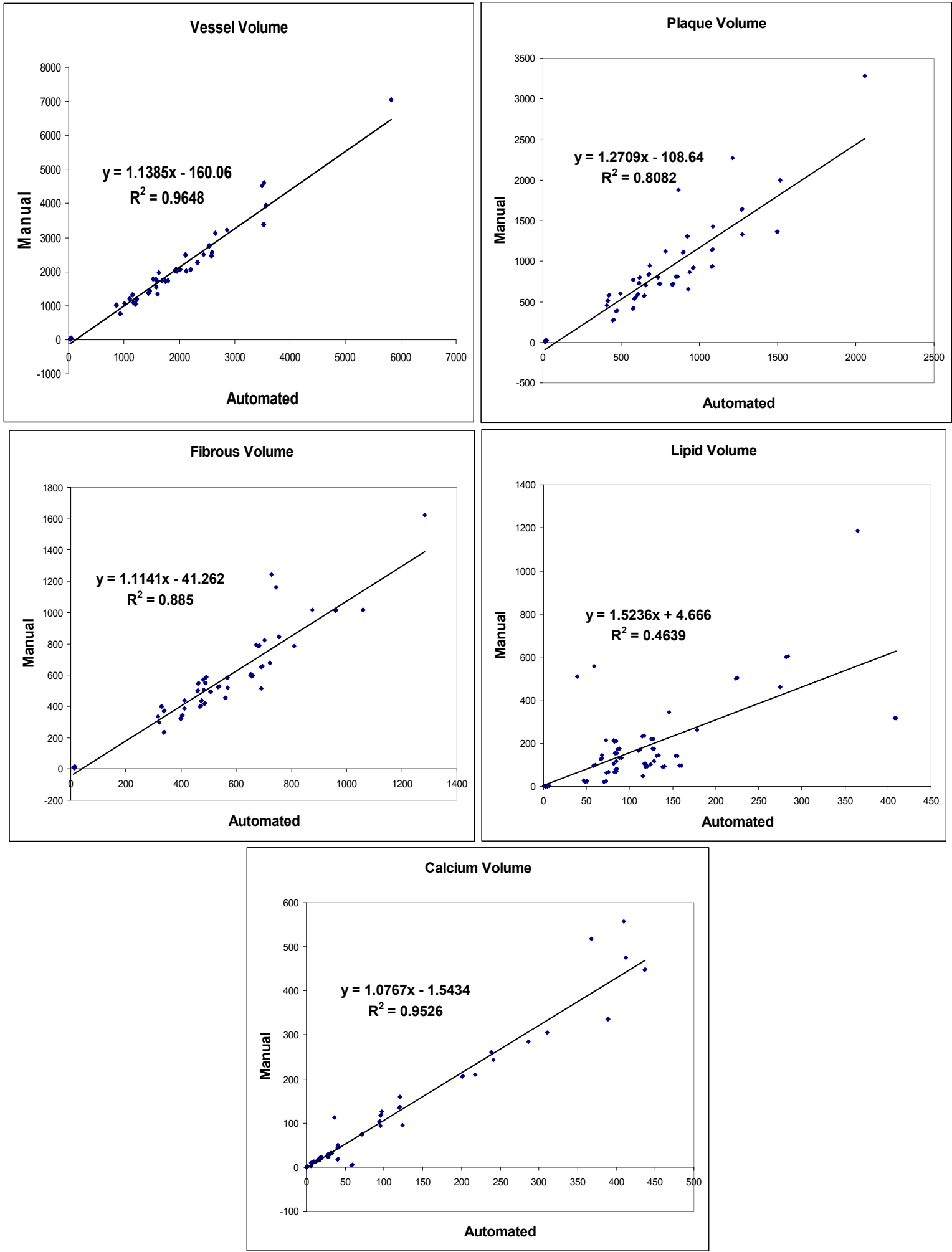

Fig. 5 Regression plots showing the comparison between automated and manual measurements on 80 datasets 
The automated levelset segmentation of the outer vessel wall was accurate, with higher average similarity index compared to method where ellipse fitting was used (a versus b; fill in values) [14].

\section{DISCUSSION \& CONCLUSION}

We presented a method to segment the outer vessel wall of the carotid bifurcation in CTA. In contrast to previous work, where we used a slice-based fixed ellipse-shaped model for the common carotid artery, we now use a levelset based segmentation, which allows segmentation of the complete bifurcation. The experiments show that the levelset based approach achieves an average Dice similarity for the vessel of $92 \%$. The method thus has large potential for the automated quantification of carotid artery plaque components.

\section{REFERENCES}

[1] World Health Organization, "Neglected Global Epidemics: three growing threats," The world health report 2003 Shaping the future, (2003).

[2] M. J. W. Koelemay, P. J. Nederkoom, J. B. Reitsma, and C. B. Majoie, "Systematic review of computed tomographic angiography for assessment of carotid artery disease," Stroke, vol. 35, no. 10, pp. 2306-2312, 2004.

[3] North American symptomatic carotid endarterectomy trial collaborators, "Beneficial effect of carotid endarterectomy in symptomatic patients with high-grade carotid stenosis," N. Engl. J. Med., vol 325, no 7, pp. 445-453, Aug. 1991

[4] S. Glagow, E. Weisenberg, C. K. Zarins, R. Stankunavicius, and G. J. Kolettis, „Compensatory enlargement of human atherosclerotic coronary arteries, “N Engl, J Med, vol 316, no. 22, pp. 1371-1375, May 1987.

[5] S. D. Olabarriaga, J. M. Rouet, M. Fradkin, M. Breeuwer and W. J. Niessen, "Segmentation of thrombus in abdominal aortic aneurysms from CTA with non-parametric statistical grey level appearance modelling," IEEE Trans. on Med. Imag., vol. 24, no.4, pp. 477 - 485, Apr. 2005.

[6] M. de Bruijne, B. van Ginneken, W. J. Niessen, M. Loog and M. A. Viergever, "Active shape model based segmentation of abdominal aortic aneurysms in CTA images," in SPIE Medical Imaging, ser. Proc. of the SPIE, vol. 4684, pp. 463-474, 2002.

[7] M. de Bruijne, B. van Ginneken, M. Viergever, and W. Niessen, "Interactive segmentation of abdominal aortic aneurysms in CTA data," Med. Image Anal., vol. 8, no. 2, pp. 127-138, 2004.

[8] M. de Bruijne, B. van Ginneken, M. Viergever, and W. Niessen, "Adapting active shape models for 3D segmentation of tubular structures in medical images," in Information Processing in Medical Imaging, ser. LNCS, vol. 2732. Springer, pp. 136-147, 2003.

[9] Yuan C, Lin E, Millard J and Hwang JN. "Closed contour edge detection of blood vessel lumen and outer wall boundaries in black-blood MR images," Magn. Reson. Imaging, vol. 17, no. 2, pp. 257-266,

Feb. 1999.

[10] G. J. Adams, G. W. Vick, C. B. Bordelon, W. Insull and J. D. Morrisett, "An algorithm for quantifying advanced carotid artery artherosclerosis in humans using MRI and active contours," Proc. SPIE Medical Imaging, vol. 4684, pp. 1448-1457, 2002.

[11] I. M. Adame, R. J. van der Geest, B. A. Wasserman, M. A. Mohamed, J. H. C. Reiber, B. P. F. Lelieveldt, "Automatic segmentation and plaque characterization in atherosclerotic carotid artery MR images," MAGMA, vol 16, no. 5, pp. 227 - 234, Apr. 2004.

[12] F. Liu, D. Xu, M. S. Ferguson, B. Chu, T. Saam, N. Takaya, T. S. Hatsukami, C. Yuan and W. S. Kerwin, "Automated in vivo segmentation of carotid plaque MRI with morphology-enhanced probability maps, " Magnetic Resonance in Medicine, vol 55, pp. 659 - 688, 2006.

[13] D.Vukadinovic, T. van Walsum, R. Manniesing, S. Rozie, R. Hameeteman, T. de Weert, A van der Lugt, W. Niessen, "Segmentation of the Outer Vessel Wall of the Common Carotid Artery in CTA", IEEE Transactions on Medical Imaging, vol 29, no 1, pp 65-76, Jan. 2010.

[14] D. Vukadinovic, T. van Walsum, S. Rozie, T. T. de Weert, R. Manniesing, A. van der Lugt and W.J. Niessen, Carotid artery segmentation and plaque quantification in CTA, IEEE International Symposium on Biomedical Imaging , 2009

[15] F. Zhuge, S. Sun, G.D. Rubin, S. Napel, "An Abdominal Aortic Aneurysm Segmentation Method: Level Set with Region and Statistical Information,” Medical Physics 33(5):1440-1453, May 2006.. 
[16] R. Manniesing, B. K. Velthuis, M. S. Van Leeuwen, I. C. Van der Schaaf, P.J. van Laar, W.J. Niessen, "Level set based cerebral vasculature segmenation and diameter quantification in CT angiography", Med. Image Anal, vol. 10, no. 2, pp. 200-214, Apr. 2006

[17] T.F. Chan and L.A. Vese, “Active Contours Without Edges", IEEE Transactions on Image Processing, vol 10, No. 2, Feb 2001.

[18] T. T. de Weert, M. Ouhlous, E. Meijering, P. E. Zondervan, J. M. Hendriks, M. R. H. M. Van Sambeek, D. W. J. Dippel, and A. van der Lugt, "In vivo characterization and quantification of atherosclerotic carotid plaque components with multidetector computed tomography and histopathological correlation," Arterioscler. Thromb. Vasc. Biol., vol. 26, no. 10, pp. 2366-2372, Oct 2006.

[19] Mosaliganti KR, Smith B, Gelas A, Gouaillard A., Megason SG "Level Set Segmentation: Active Contours Without Edges". Insight Journal, 2009, http://www.insight-journal.org/browse/publication/322

\section{ORIGINAL SUBMISSION}

This work is original and has not been submitted elsewhere. 